\title{
Discrete Approximations for Multidimensional Singular Integral Operators
}

\author{
Alexander Vasilyev $^{1,2}$ and Vladimir Vasilyev ${ }^{1,2(\bowtie)}$ \\ 1 Department of Mathematical Analysis, National Research Belgorod State \\ University, Studencheskaya 14/1, 308007 Belgorod, Russia \\ alexvassel@gmail.com, vbv57@inbox.ru \\ 2 Chair of Pure Mathematics, Lipetsk State Technical University, \\ Moskovskaya 30, 398600 Lipetsk, Russia
}

\begin{abstract}
For discrete operator generated by singular kernel of Calderon-Zygmund one introduces a finite dimensional approximation which is a cyclic convolution. Using properties of a discrete Fourier transform and a finite discrete Fourier transform we prove a solvability for approximating equation in corresponding discrete space. For comparison discrete and finite discrete solution we obtain an estimate for a speed of convergence for a certain right-hand side of considered equation.
\end{abstract}

Keywords: Discrete Calderon-Zygmund operator · Discrete fourier transform $\cdot$ Cyclic convolution $\cdot$ Approximation rate

\section{Introduction}

A basic object of this paper is a multidimensional singular integral

$$
v . p . \int_{D} K(x, x-y) u(y) d y, \quad x \in D,
$$

which generates the Calderon-Zygmund operator with variable kernel $[1,2]$, where $D$ is a domain in $\mathbb{R}^{m}$ unbounded as a rule.

Taking into account forthcoming studies of such a general operator using a local principle here we consider a case of constant coefficients, i.e. when the kernel does not depend on a first variable and the Calderon-Zygmund operator looks as follows

$$
\begin{gathered}
(K u)(x) \equiv v \cdot p \cdot \int_{D} K(x-y) u(y) d y \equiv \\
\lim _{\varepsilon \rightarrow 0} \int_{D \cap\{|x-y|>\varepsilon\}} K(x-y) u(y) d y, x \in D .
\end{gathered}
$$

We assume here that the kernel $K(x)$ of the integral (1) satisfy the following conditions: (1) $K(x)$ is a homogeneous function of order $-m, K(t x)-$ $t^{-m} K(x), \forall t>0 ;(2) K(x)$ is a differentiable function on a unit sphere $S^{m-1}$; (3) the function $K(x)$ has zero mean value on the $S^{m-1}[1,2]$. 


\subsection{Canonical Domains}

We will consider different types of domains $D$ because the theory is essentially depended on this type. So for example cases $D=\mathbb{R}^{m}$ and $D=\mathbb{R}_{+}^{m}=\left\{x \in \mathbb{R}^{m}\right.$ : $x=\left(x_{1}, \cdots, x_{m}, x_{m}>0\right\}$ are essentially distinct. Invertibility conditions for the operator $K$ do not coincide for these cases.

\subsection{Infinite Discrete Case-Series}

To obtain a good approximation for the integral (1) we will use the following reduction. First instead of the integral (1) we introduce the series

$$
\sum_{\tilde{y} \in D \cap h \mathbb{Z}^{m}} K(\tilde{x}-\tilde{y}) u_{d}(\tilde{y}) h^{m},
$$

which generates a discrete operator $K_{d}$ defined on functions $u_{d}$ of discrete variable $\tilde{x} \in D \cap h \mathbb{Z}^{m}$. Since the Calderon-Zygmund kernel has a strong singularity at the origin we mean $K(0)=0$. Convergence for the series (2) means that the following limit

$$
\lim _{N \rightarrow+\infty} \sum_{\tilde{y} \in h \mathbb{Z}^{m} \cap Q_{N} \cap D} K(\tilde{x}-\tilde{y}) u_{d}(\tilde{y}) h^{m}
$$

exists, where $Q_{N}=\left\{x \in \mathbb{R}^{m}: \max _{1 \leq k \leq m}\left|x_{k}\right|<N\right\}$. It was shown earlier that a norm of the operator $K_{d}: L_{2}\left(h \overline{\mathbb{Z}}^{m} \cap D\right) \rightarrow L_{2}\left(h \mathbb{Z}^{m} \cap D\right)$ does not depend on $h$ [9]. But although the operator is a discrete object it is an infinite one, and to solve equations with a such operator one needs to replace it by a certain finite object.

\subsection{Finite Discrete Case-Systems of Linear Algebraic Equations}

It is natural to consider a system of linear algebraic equation instead of an infinite system generated by the series (2). This method is called projection method [3]. It proposes following actions. Let $P_{N}: L_{2}\left(D \cap \mathbb{Z}^{m}\right) \rightarrow L_{2}\left(D \cap Q_{N} \cap \mathbb{Z}^{m}\right)$ be a projector on a finite dimensional space. One needs to solve the equation

$$
P_{N} K_{d} P_{N} u_{d}=P_{N} v_{d}
$$

in the space $L_{2}\left(D \cap Q_{N} \cap \mathbb{Z}^{m}\right)$ instead of the infinite system of linear algebraic equations

$$
K_{d} u_{d}=v_{d}
$$

in the space $L_{2}\left(D \cap \mathbb{Z}^{m}\right)$.

The following result plays a key role in the theory of projection methods, and it was proved for some special domains $D$ and integrable kernel $K(x)$ [3].

Proposition 1. If the Eq. (4) is uniquely solvable in the space $L_{2}\left(D \cap \mathbb{Z}^{m}\right)$ for arbitrary right-hand side $v_{d} \in L_{2}\left(D \cap \mathbb{Z}^{m}\right)$ then the Eq. (3) is uniquely solvable in the space $L_{2}\left(D \cap Q_{N} \cap \mathbb{Z}^{m}\right)$ for enough large $N$. 
Here we consider the case $D=\mathbb{R}^{m}$ and prove this proposition 1 with some novelties because the Eq.(4) is a system of linear algebraic equations and for large $N$ one needs much time to solve it.

Our considerations are based on two steps: continual infinite object $(1) \longrightarrow$ discrete infinite object $(2) \longrightarrow$ discrete finite object (3) with justification and error estimates. Some pieces of this programm were realized in authors' papers $[4-9]$.

\section{Discrete Fourier Transform and Symbols}

Let us define the discrete Fourier transform for functions $u_{d}$ of a discrete variable $\tilde{x} \in h \mathbb{Z}^{m}$

$$
\left(F_{d} u_{d}\right)(\xi)=\sum_{\tilde{x} \in h \mathbb{Z}^{m}} u_{d}(\tilde{x}) e^{i \tilde{x} \cdot \xi} h^{m}, \quad \xi \in \hbar \mathbb{T}^{m}, \hbar=\frac{h^{-1}}{2 \pi},
$$

where $\mathbb{T}^{m}$ is $m$-dimensional cube $[-\pi, \pi]^{m}$.

Such discrete Fourier transform preserves all basic properties of the classical fourier transform, particularly for a discrete convolution of two discrete functions $u_{d}, v_{d}$

$$
\left(u_{d} * v_{d}\right)(\tilde{x}) \equiv \sum_{\tilde{y} \in h \mathbb{Z}^{m}} u_{d}(\tilde{x}-\tilde{y}) v_{d}(\tilde{y}) h^{m}
$$

we have the well known multiplication property

$$
\left(F_{d}\left(u_{d} * v_{d}\right)\right)(\xi)=\left(F_{d} u_{d}\right)(\xi) \cdot\left(F_{d} v_{d}\right)(\xi) .
$$

If we apply this property to the operator $K_{d}$ we obtain

$$
\left(F_{d}\left(K_{d} u_{d}\right)\right)(\xi)=\left(F_{d} K_{d}\right)(\xi) \cdot\left(F_{d} u_{d}\right)(\xi) .
$$

Let us denote $\left(F_{d} K_{d}\right)(\xi) \equiv \sigma_{d}(\xi)$ and give the following

Definition 1. The function $\sigma_{d}(\xi), \xi \in \hbar \mathbb{T}^{m}$, is called a periodic symbol of the operator $K_{d}$.

We will assume below that the symbol $\sigma_{d}(\xi) \in C\left(\hbar \mathbb{T}^{m}\right)$ therefore we have immediately the following

Property 1. The operator $K_{d}$ is invertible in the space $L_{2}\left(h \mathbb{Z}^{m}\right)$ iff $\sigma_{d}(\xi) \neq 0, \forall \xi \in \hbar \mathbb{T}^{m}$.

Definition 2. A continuous periodic symbol is called an elliptic symbol if $\sigma_{d}(\xi) \neq 0, \forall \xi \in \hbar \mathbb{T}^{m}$.

So we see that an arbitrary elliptic periodic symbol $\sigma_{d}(\xi)$ corresponds to an invertible operator $K_{d}$ in the space $L_{2}\left(h \mathbb{Z}^{m}\right)$.

Remark 1. It was proved earlier that operators (1) and (2) for cases $D=$ $\mathbb{R}^{m}, D=\mathbb{R}_{+}^{m}$ are invertible or non-invertible in spaces $L_{2}\left(\mathbb{R}^{m}\right), L_{2}\left(\mathbb{R}_{+}^{m}\right)$ and $L_{2}\left(h \mathbb{Z}^{m}\right), L_{2}\left(h \mathbb{Z}_{+}^{m}\right)$ simultaneously $[6,8]$. 


\section{Periodic Approximation and Cyclic Convolutions}

Here we will introduce a special discrete periodic kernel $K_{d, N}(\tilde{x})$ which is defined as follows. We take a restriction of the discrete kernel $K_{d}(\tilde{x})$ on the set $Q_{N} \cap \mathbb{Z}^{m} \equiv$ $Q_{N}^{d}$ and periodically continue it to a whole $\mathbb{Z}^{m}$. Further we consider discrete periodic functions $u_{d, N}$ with discrete cube of periods $Q_{N}^{d}$. We can define a cyclic convolution for a pair of such functions $u_{d, N}, v_{d, N}$ by the formula

$$
\left(u_{d, N} * v_{d, N}\right)(\tilde{x})=\sum_{\tilde{y} \in Q_{N}^{d}} u_{d, N}(\tilde{x}-\tilde{y}) v_{d, N}(\tilde{y}) h^{m} .
$$

(We would like to remind that such convolutions are used in digital signal processing [10]). Further we introduce finite discrete Fourier transform by the formula

$$
\left(F_{d, N} u_{d, N}\right)(\tilde{\xi})=\sum_{\tilde{x} \in Q_{N}^{d}} u_{d, N}(\tilde{x}) e^{i \tilde{x} \cdot \tilde{\xi}} h^{m}, \quad \tilde{\xi} \in R_{N}^{d}
$$

where $R_{N}^{d}=\hbar \mathbb{T}^{m} \cap \hbar \mathbb{Z}^{m}$. Let us note that here $\tilde{\xi}$ is a discrete variable.

According to the formula (5) one can introduce the operator

$$
K_{d, N} u_{d, N}(\tilde{x})=\sum_{\tilde{y} \in Q_{N}^{d}} K_{d, N}(\tilde{x}-\tilde{y}) u_{d, N}(\tilde{y}) h^{m}
$$

on periodic discrete functions $u_{d, N}$ and a finite discrete Fourier transform for its kernel

$$
\sigma_{d, N}(\tilde{\xi})=\sum_{\tilde{x} \in Q_{N}^{d}} K_{d, N}(\tilde{x}) e^{i \tilde{x} \cdot \tilde{\xi}} h^{m}, \quad \tilde{\xi} \in R_{N}^{d} .
$$

Definition 3. A function $\sigma_{d, N}(\tilde{\xi}), \tilde{\xi} \in R_{N}^{d}$, is called s symbol of the operator $K_{d, N}$. This symbol is called an elliptic symbol if $\sigma_{d, N}(\tilde{\xi}) \neq 0, \forall \tilde{\xi} \in R_{N}^{d}$.

Theorem 1. Let $\sigma_{d}(\xi)$ be an elliptic symbol. Then for enough large $N$ the symbol $\sigma_{d, N}(\tilde{\xi})$ is elliptic symbol also.

Proof. The function

$$
\sum_{\tilde{x} \in Q_{N}^{d}} K_{d, N}(\tilde{x}) e^{i \tilde{x} \cdot \xi} h^{m}, \quad \xi \in \hbar \mathbb{T}^{m},
$$

is a segment of the Fourier series

$$
\sum_{\tilde{x} \in h \mathbb{Z}^{m}} K_{d}(\tilde{x}) e^{i \tilde{x} \cdot \xi} h^{m}, \quad \xi \in \hbar \mathbb{T}^{m},
$$

and according our assumptions this is continuous function on $\hbar \mathbb{T}^{m}$. Therefore values of the partial sum coincide with values of $\sigma_{d, N}$ in points $\tilde{\xi} \in R_{N}^{d}$. Besides these partial sums are continuous functions on $\hbar \mathbb{T}^{m}$.

As before an elliptic symbol $\sigma_{d, N}(\tilde{\xi})$ corresponds to the invertible operator $K_{d, N}$ in the space $L_{2}\left(Q_{N}^{d}\right)$. 


\section{Approximation Rate}

Let $A: B \rightarrow B$ be a linear bounded operator acting in a Banach space $B$, $B_{N} \subset B$ be its finite dimensional subspace, $P_{N}: B \rightarrow B_{N}$ be a projector, $A_{N}: B_{N} \rightarrow B_{N}$ linear finite-dimensional operator [5].

Definition 4. Approximation rate for operators $A$ and $A_{N}$ is called the following operator norm

$$
\left\|P_{N} A-A_{N} P_{N}\right\|_{B \rightarrow B_{N}}
$$

We will obtain a "weak estimate" for approximation rate but enough for our purposes. We assume additionally that a function $u_{d}$ is a restriction on $h \mathbb{Z}^{m}$ of continuous function with certain estimates $[4,5]$. Let's define the discrete space $C_{h}(\alpha, \beta)$ as a functional space of discrete variable $\tilde{x} \in h \mathbb{Z}^{m}$ with finite norm

$$
\left\|u_{d}\right\|_{C_{h}(\alpha, \beta)}=\left\|u_{d}\right\|_{C_{h}}+\sup _{\tilde{x}, \tilde{y} \in h \mathbb{Z}^{m}} \frac{|\tilde{x}-\tilde{y}|^{\alpha}}{(\max \{1+|\tilde{x}|, 1+|\tilde{y}|\})^{\beta}} .
$$

It means that the function $u_{d} \in C_{h}(\alpha, \beta)$ satisfies the following estimates

$$
\begin{gathered}
\left|u_{d}(\tilde{x})-u_{d}(\tilde{y})\right| \leq c \frac{|\tilde{x}-\tilde{y}|^{\alpha}}{(\max \{1+|\tilde{x}|, 1+|\tilde{y}|\})^{\beta}}, \\
\left|u_{d}(\tilde{x})\right| \leq \frac{c}{(1+|\tilde{x}|)^{\beta-\alpha}}, \quad \forall \tilde{x}, \tilde{y} \in h \mathbb{Z}^{m}, \alpha, \beta>0,0<\alpha<1 .
\end{gathered}
$$

Let us note that under required assumptions $C_{h}(\alpha, \beta) \subset L_{2}\left(h \mathbb{Z}^{m}\right)$.

Theorem 2. For operators $K_{d}$ and $K_{d, N}$ we have the following estimate

$$
\left\|\left(P_{N} K_{d}-K_{d, N} P_{N}\right) u_{d}\right\|_{L_{2}\left(Q_{N}^{d}\right)} \leq C N^{m+2(\alpha-\beta)}
$$

for arbitrary $u_{d} \in C_{h}(\alpha, \beta), \beta>\alpha+m / 2$.

Proof. Let us write

$$
\left(P_{N} K_{d}-K_{d, N} P_{N}\right) u_{d}=P_{N} K_{d} P_{N} u_{d}-K_{d, N} P_{N} u_{d}+P_{N} K_{d}\left(I-P_{N}\right) u_{d},
$$

where $I$ is an identity operator in $L_{2}\left(h \mathbb{Z}^{m}\right)$.

First two summands have annihilated, and we need to estimate only the last summand. We have

$$
\left\|P_{N} K_{d}\left(I-P_{N}\right) u_{d}\right\| \leq C\left\|\left(I-P_{N}\right) u_{d}\right\|
$$

because norms of operators $K_{d}$ are uniformly bounded, and for the last norm taking into account (6) we can write

$$
\left\|\left(I-P_{N}\right) u_{d}\right\|^{2} \leq C \sum_{\tilde{x} \in h \mathbb{Z}^{m} \backslash Q_{N}}\left|u_{d}(\tilde{x})\right|^{2} h^{m} \leq C \sum_{\tilde{x} \in h \mathbb{Z}^{m} \backslash Q_{N}} \frac{h^{m}}{(1+|\tilde{x}|)^{2(\beta-\alpha)}} \leq
$$

and further

$$
C \int_{\mathbb{R}^{m} \backslash Q_{N}}|x|^{2(\alpha-\beta)} d x
$$

The last integral using spherical coordinates gives the estimate $N^{m+2(\alpha-\beta)}$ which tends to 0 under $n \rightarrow \infty$ if $\beta>\alpha+m / 2$. 


\section{Main Theorem on Approximation}

Here we consider the equation

$$
K_{d, N} u_{d, N}=P_{N} v_{d}
$$

instead of the Eq. (4) and give a comparison for these two solutions.

Below we assume that operator $K_{d}$ is invertible in $L_{2}\left(h \mathbb{Z}^{m}\right)$.

Theorem 3. If $v_{d} \in C_{h}(\alpha, \beta), \beta>\alpha+m / 2, u_{d}$ is a solution of the Eq. (4), $u_{d, N}$ is a solution of (7) then the estimate

$$
\left\|u_{d}-u_{d, N}\right\|_{L_{2}\left(h \mathbb{Z}^{m}\right)} \leq C N^{m+2(\alpha-\beta)}
$$

is valid, and $C$ is a constant non-depending on $N$.

Proof. Let us write

$$
\begin{gathered}
u_{d}-u_{d, N}=K_{d}^{-1} v_{d}-K_{d, N}^{-1} P_{N} v_{d}= \\
\left(I-P_{N}\right) K_{d}^{-1} v_{d}+P_{N} K_{d}^{-1} v_{d}-K_{d, N}^{-1} P_{N} v_{d}
\end{gathered}
$$

For the summand $P_{N} K_{d}^{-1} v_{d}-K_{d, N}^{-1} P_{N} v_{d}$ we have a corresponding estimate by the Theorem 2 because the operators $K_{d}^{-1}$ and $K_{d, N}^{-1}$ are constructed similar initial operators $K_{d}$ and $K_{d, N}$.

The first summand is estimated like the proof of the Theorem 2 and using the property that operator $K_{d}$ is uniformly on $h$ is bounded in the space $C_{h}(\alpha, \beta)$ [9] and the operator $K_{d}^{-1}$ has a symbol with required properties [8].

\section{Conclusion}

We have introduced such a finite approximation for original integral (1) because there are a lot of algorithms for calculating a finite discrete Fourier transform, these are so called fast Fourier transform algorithms [10]. On the other hand this step-by-step approximation permits to justify mathematically without additional difficulties results on a solvability for a corresponding approximate equation.

Acknowledgments. The work was supported by Russian Foundation for Basic Research and Lipetsk regional government of Russia, project No. 14-41-03595-rcenter-a.

\section{References}

1. Selected papers of Alberto P. Calderon with commentary. In: Bellow, A., Kenig, C.E., Malliavin, P. (eds.) AMS, Providence (2008)

2. Mikhlin, S.G., Prößdorf, S.: Singular Integral Operators. Akademie-Verlag, Berlin (1986) 
3. Gohberg, I.C., Feldman, I.A.: Convolution Equations and Projection Methods for Their Solution. AMS, Providence (1974)

4. Vasilyev, A.V., Vasilyev, V.B.: Numerical analysis for some singular integral equations. Neural, Parallel, Sci. Comput. 20, 313-326 (2012)

5. Vasilyev, A.V., Vasilyev, V.B.: Approximation rate and invertibility for some singular integral operators. Proc. Appl. Math. Mech. 13, 373-374 (2013)

6. Vasilyev, A.V., Vasilyev, V.B.: Discrete singular operators and equations in a halfspace. Azerb. J. Math. 3, 84-93 (2013)

7. Vasil'ev, A.V., Vasil'ev, V.B.: Periodic Riemann problem and discrete convolution equations. Diff. Equat. 51, 652-660 (2015)

8. Vasilyev, A.V., Vasilyev, V.B.: Discrete singular integrals in a half-space. In: Mityushev, V., Ruzhansky, M. (eds.) Current Trends in Analysis and Its Applications. Trends in Mathematics. Research Perspectives, pp. 663-670. Birkhäuser, Basel (2015)

9. Vasilyev, A.V., Vasilyev, V.B.: On the solvability of certain discrete equations and related estimates of discrete operators. Dokl. Math. 92, 585-589 (2015)

10. Oppenheim, A.V., Schafer, R.W.: Digital Signal Processing. Prentice Hall, Englewood Cliffs (1975) 